\title{
Stenotrophomonas maltophilia Keratitis Related to Therapeutic Contact Lens Misidentified with an Automated Identification System
}

\author{
Daniel Fatela-Cantillo1* ${ }^{*}$, Javier Crespo-González², Blanca Fatela-Cantillo3, \\ Antonio Fernandez-Suarez ${ }^{1}$, Jose Miguel Aguilar Benítez ${ }^{4}$, \\ Juan Antonio Saez Nieto5, Remedios González-Amezcua ${ }^{6}$ \\ ${ }^{1}$ Department of Biotechnology, Hospital Alto Guadalquivir, Andújar (Jaén), Spain \\ ${ }^{2}$ Department of Ophthalmology, Hospital Linares, Linares (Jaén), Spain \\ ${ }^{3}$ Department of Ophthalmology, Hospital La Princesa, Madrid (Madrid), Spain \\ ${ }^{4}$ Department of Biotechnology, Hospital Alcalá La Real, Alcalá La Real (Jaén), Spain \\ ${ }^{5}$ NationalMicrobiology Centre, Majadahonda (Madrid), Spain \\ ${ }^{6}$ Department of Ophthalmology, Hospital Alto Guadalquivir, Andújar (Jaén), Spain

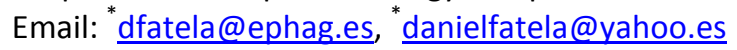

Received 27 March 2015; accepted 26 May 2015; published 29 May 2015

Copyright (C) 2015 by authors and Scientific Research Publishing Inc.

This work is licensed under the Creative Commons Attribution International License (CC BY).

http://creativecommons.org/licenses/by/4.0/

(c) (i) Open Access

\begin{abstract}
We present a case of keratitis caused by Stenotrophomonas maltophilia in a therapeutic contact lens user with trichiasis and symblepharon. This keratitis was initially diagnosed as caused by Achromobacter xylosoxidans, but the strain was sent for species confirmation and the isolate was finally identified as $S$. maltophilia by means of $16 \mathrm{~S}$ rDNA sequencing. The patient rapidly improved on administration of fortified ceftazidime. Physicians should be aware that the definitive identification of the pathogenic agent and prolonged antimicrobial treatment according to culture sensitivities in keratitis are mandatory as treatment success depends greatly on them.
\end{abstract}

Keywords

Stenotrophomonas maltophilia, Keratitis, Ceftazidime

${ }^{*}$ Corresponding author.

How to cite this paper: Fatela-Cantillo, D., et al. (2015) Stenotrophomonas maltophilia Keratitis Related to Therapeutic Contact Lens Misidentified with an Automated Identification System. Open Journal of Clinical Diagnostics, 5, 63-67.

http://dx.doi.org/10.4236/ojcd.2015.52012 


\section{Introduction}

Stenotrophomonas maltophilia is a motile, ubiquitous and aerobic Gram-negative opportunistic organism that had been seldom-reported as a cause of ocular infection in the past. However, currently it is being noted as an emerging nosocomial pathogen that is difficult to treat due to multiple drug resistance and which can cause potentially life-threatening infections in the immunocompromised. It has been published that it is resistant to many antibiotics due to the production of two enzymes (L1 and L2) and its poor outer membrane permeability. The treatment combination of choice is trimethoprim-sulphamethoxazole, in spite of being bacteriostatic [1].

It can be involved in several ophthalmologic conditions such as keratitis, conjunctivitis, dacryocystitis and cellulitis [2]. Most infections by this organism occur in patients with ocular compromise. Such is the case of the patient we are about to describe herein.

\section{Case Report}

81-year-old male patient with history of hospitalizations due to a chronic heart condition, who came into our ophthalmologic service with a 6-day history of pain in his right eye (RE). The patient was using a therapeutic contact lens (TCL) in his RE due to recurrent corneal ulcerations secondary to trichiasis.

The patient underwent cataract surgery in both eyes several years ago, with a well tolerated anterior chamber lens in the RE. Over the years, the patient developed chronic compromise to the ocular surface secondary to the use of hypotensive eye drops, resulting in trichiasis and symblepharon. Trichiasis happens when eyelashes grow from abnormal spots on the eyelid; this can cause secondary corneal ulceration, while symblepharon is the partial or complete adhesion of the palpebral conjunctiva of the eyelid to the bulbar conjunctiva of the eyeball that basically occurs as a consequence of abnormal scarring processes.

On examination, the patient's visual acuity was "counting fingers" with his RE and 8/10 with his left eye (LE). Slit-lamp examination revealed a corneal stromal infiltrate with an underlying epithelial defect inferior to the visual axis in the RE; LE showed no abnormalities. The patient was immediately ordered to stop using the TCL and scrapings from the cornea were collected for culture. The treatment regimen started with cyclopentolate, autologous serum eye drops, and hourly $0.5 \%$ moxifloxacin.

Five days after the first visit, the RE cornea was scraped with a sterile corneal spatula, and samples were sent for fungal, viral, bacterial and protozoal cultures. On non selective culture media, colonies of an aerobic gramnegative bacillus grew. The bacteria appeared rough and nonpigmented at 48 hours on the MacConkey agar. Achromobacter xylosoxidans subsp. xylosoxidans was determined using a conventional panel of classical biochemical substrates (MicroScan-Neg Type 53; Siemens) in an automated identification (ID) system (MicroScan Walk Away [W/A]; Siemens); the results of drug sensitivity tests resulted positive to trimethoprim/sulfametoxazol, piperaciline/tazobactam and ceftazidime. We identified the isolate as resistant to ciprofloxacin. All W/A susceptibility interpretations are shown in Table 1 using Clinical and Laboratory Standards Institute (CLSI) for MIC interpretative criteria.

Table 1. Antimicrobial susceptibilities.

\begin{tabular}{cc}
\hline Antibiotic & MIC $(\mathrm{mcg} / \mathrm{mL}) /$ Interpretation \\
\hline Amikacin & $>32 / \mathrm{R}$ \\
Trimethoprim/sulfametoxazol & $>\mathbf{2} / \mathbf{3 8 / S}$ \\
Cefepime & $>16 / \mathrm{R}$ \\
Cefotaxime & $>32 / \mathrm{R}$ \\
Ciprofloxacin & $>2 / \mathrm{R}$ \\
Gentamicin & $>8 / \mathrm{R}$ \\
Tobramycin & $>8 / \mathrm{R}$ \\
Imipenem & $>8 / \mathrm{R}$ \\
Piperacilin/tazobactam & $>\mathbf{6 4 / S}$ \\
Ceftazidime & $>\mathbf{1 6 / S}$ \\
\hline
\end{tabular}

R: resistant; S: sensitive. 
We suspected a W/A misidentification due to discrepancies on oxidase reaction (Biomerieux; France). If we selected a negative result for that test and the non-fermentation of glucose and maltose the ID was different, with a 99.9\% of probability for Stenotrophomonas maltophilia in W/A results. As there was no DNAase culture medium available in our hospital area the strain was sent to the National Centre for Microbiology for species confirmation and the isolate was identified as S. maltophilia by means of BIOLOG GN2 pannel (BIOLOG Inc. Hayward, USA) with 95 carbon sources. We found a similarity of $99.8 \% \mathrm{~T}=0.698$ with this species. The identification was confirmed by means of $16 \mathrm{~s}$ rRNA gene sequence analysis. A fragment of $1450 \mathrm{bp}$ was obtained by the PCR method previously described [3]. The sequence showed a 99.7\% homology with S. maltophilia from the Gene Bank (accession number GQ360071 and others). Currently, because several previously proposed species are recognized to be closely related to $S$. maltophilia, it might be referred to as S. maltophilia complex [4].

In our strain of S. maltophilia the ceftazidime minimum inhibitory concentration (MIC) with E-test was 12 $\mathrm{mcg} / \mathrm{mL}$. According to these results, moxifloxacin treatment was discontinued and hourly fortified ceftazidime eye drops were introduced in the treatment. During the examination, it was noted that the visual acuity in the patient's RE had improved to $1 / 10$ and the corneal epithelium had healed, while the stroma still showed mild opacities.

One month later, the patient was still using fortified ceftazidime eye drops every 3 hours during daytime, and his visual acuity reached 4/10 with his RE. Pictures of the cornea were taken, under the patient's agreement for further scientific use, and showed a complete resolution of the infiltrate with slight corneal scarring, which meant that the patient couldn't gain his previous best corrected visual acuity of 5/10 with his RE.

A new culture of the cornea was performed at this time and antibiotic treatment was discontinued as soon as the negative result of the culture was available. The patient was advised to continue using autologous serum eye drops and, after 7 months of follow-up, keratitis did not recur, keeping the same visual acuity of 4/10.

\section{Discussion}

Stenotrophomonas maltophilia, which was previously designated as Pseudomonas maltophilia and Xanthomonas maltophilia, is an emerging multidrug-resistant global opportunistic pathogen [5]. The most frequent clinical manifestations of $S$. maltophilia infection are bloodstream infections and pneumonia. The S. maltophilia bacteremia is typically central venous catheter-related. Other clinical manifestations to $S$. maltophilia infections include endocarditis, endophtalmitis, sinusitis, cellulitis, meningitis, liver abcess, and myositis, but several reports have shown ocular involvement in patients with ocular conditions such as trauma, surgery or soft contact lens use [6].

This report provides clinical evidence of keratitis caused by S. maltophilia in a user of therapeutic contact lens which was initially misidentified as A. xylosoxidans subsp. xilosoxidans, based on a routine automated ID method. A. xylosoxidans is a gram-negative bacillus that belongs to the genus Alcaligenes which has a widespread distribution worldwide [7]. As mentioned before, most ocular infections by S. maltophilia occur in patients with ocular compromise. However, it is a seldom-reported bacteria in lens care systems [6].

S. maltophilia is a motile, non-fermentative, oxidase-negative, aerobic, gram-negative bacillus. S. maltophilia grows readily on most bacteriologic media, typically appearing pale-yellow, grayish or lavender-green when grown on blood agar. The organism can be commonly found in water and may be confused with Pseudomonas species and other gram-negative bacillus such as A. xylososidans. However, the identification of S. maltophilia does not usually cause problems using rapid automated identification systems and is usually accompanied by a characteristically resistant antibiogram, although it is known that W/A system has sometimes considerable difficulty in identifying unusual isolates that are occasionally recovered. Some investigators have emphasized the need of more robust tests, using more reliable phenotypic or genotypic methods, rather than only using these automated systems for the ID of unusual nonfermentative gram-negative bacilli and other bacteria that are difficult to identify with phenotypic schemes commonly used outside reference laboratories [3] [8].

Reports of postoperative and post-traumatic $S$. maltophilia ocular infections are increasing and the major predisposing factor of S. maltophilia infection is prior exposure to antibiotics [9]. It is difficult to treat ocular infections caused by S. maltophilia because of shortage of eye drops of antibiotics useful to treat this bacterium and the low concentration of antibiotics reached in the eyeball during treatment. Most ocular isolates of S. maltophilia were resistant to aminoglycosides and most beta-lactams, and showed variable susceptibility to quinolones [2]. Since the pathogen is resistant to many antibiotics, it is important to select an appropriate therapeutic agent. 
Other reports have evaluated monotherapy with fluoroquinolone eye drops for the treatment of bacterial corneal ulcers with discrepancies on results and recommendations of caution when using fluoroquinolones in large, deep eye ulcers in the elderly [10] [11].

Culture dependent methodologies identified Pseudomonas aeruginosa as the most common pathogen in contact lens-related infections; however, another study showed that Achromobacter, Stenotrophomonas, and Delftia were predominant bacteria, drawing attention to their emerging role in contact lens-related keratitis and the association between disease severity and bacterial diversity in biofilms isolated from cases and lenses of patients with contact lens-related corneal disease [12]. The possibility of biofilm formation in this case and the capacity of colonization of abiotic surfaces such as contact lens by S. maltophilia is an important characteristic of this bacterium.

In this patient moxifloxacin was used at first, until we considered antibiotic susceptibility studies and fortified ceftazidime was selected as the most appropriate treatment. The agent with best documented clinical activity in our case was trimethoprim-sulfamethoxazole, and this is the only agent for which EUCAST breakpoints are currently available (susceptible $\leq 4 \mathrm{mg} / \mathrm{L}$; resistant $>4 \mathrm{mg} / \mathrm{L}$ ). Despite our best efforts, our Pharmacy department could not supply us with fortified trimethoprim-sulfamethoxazole eye drops on time, as this medication is not commercially available in Spain. Therefore, as the patient needed prompt treatment we chose fortified ceftazidime instead. Although the Clinical and Laboratory Standards Institute (CLSI) has validated minocycline, levofloxacin and trimethoprim-sulfamethoxazole for disk-diffusion and, the same antibiotics and ticarcillincalvulanic acid, ceftazidime and cloramphenicol for MIC interpretative criteria. Fortunately, our patient's keratitis rapidly improved on administration of fortified ceftazidime.

\section{Conclusion}

In conclusion, we describe a case of keratitis caused by S. maltophilia, which automated tests misidentified as $A$. xylosoxidans subsp. xylosoxidans. There appears to be a need for a system that can readily distinguish $S$. maltophilia from A. xylososidans subsp. xylosoxidans in local clinical microbiology laboratories. The pathogenicity of $S$. maltophilia in keratitis should be fully investigated because inaccurate characterization and treatment of the pathogen may result in prolonged infection, permanent damage to ocular tissues and finally, visual impairment.

\section{References}

[1] Denton, M. and Kerr, K.G. (1998) Microbiological and Clinical Aspects of Infection Associated with Stenotrophomonas maltophilia. Clinical Microbiology Reviews, 2, 57-80.

[2] Penland, R.L. and Wilhemus, K.R. (1996) Stenotrophomonas maltophilia Ocular Infections. Archives of Ophthalmology, 114, 433-436. http://dx.doi.org/10.1001/archopht.1996.01100130429013

[3] Drancourt, M., Bollet, C., Carlioz, A., Martelin, R., Gayralt, J.P. and Raoult, D. (2000) 16s Ribosomal DNA Sequence Analysis of a Large Collection of Environmental and Clinical Unidentified Bacterial Isolates. Journal of Clinical Microbiology, 38, 2623-2630.

[4] Rhee, J.-Y., Choi, J.Y., Choi, M.-J., Song, J.-H., Peck, K.R. and Koo, K.S. (2013) Distinct Groups and Antimicrobial Resistance of Clinical Stenotrophomonas maltophilia Complex Isolates from Korea. Journal of Medical Microbiology, 62, 748-753. http://dx.doi.org/10.1099/jmm.0.053355-0

[5] Brooke, J.S. (2012).Stenotrophomonas maltophilia: An Emerging Global Opportunistic Pathogen. Clinical Microbiology Reviews, 25, 2-41. http://dx.doi.org/10.1128/CMR.00019-11

[6] Neumaier-Ammerer, B., Stolba, U., Feichtinger, H. and Binder, S. (2008) Contact Lens Related Corneal Infiltrates and Ulcers-A Retrospective Study of 134 Eyes. Spektrum der Augenheilkunde., 22, 297-300. http://dx.doi.org/10.1007/s00717-008-0284-7

[7] Park, J.H., Song, N.H. and Koh, J.W. (2012) Achromobacter xylosoxidans Keratitis after Contact Lens Usage. Korean Journal of Ophthalmology, 26, 49-53. http://dx.doi.org/10.3341/kjo.2012.26.1.49

[8] Snyder, J.W., Munier, G.K. and Johnson, C.L. (2008) Direct Comparison of the BD Phoenix System with the MicroScanWalkAway System for Identification and Antimicrobial Susceptibility Testing of Enterobacteriaceae and Nonfermentative Gram-Negative Organisms. Journal of Clinical Microbiology, 46, 2327-2333. http://dx.doi.org/10.1128/JCM.00075-08

[9] Son, S.W., Kim, H.J. and Seo, J.W. (2011) A Case of Stenotrophomonas maltophilia Keratitis Effectively Treated with Moxifloxacin. Korean Journal of Ophthalmology, 25, 349-351. http://dx.doi.org/10.3341/kjo.2011.25.5.349 
[10] Gangopadhyay, N., Daniell, M., Weih, L. and Taylor, H.R. (2000) Fluoroquinolone and Fortified Antibiotics for Treating Bacterial Corneal Ulcers. British Journal of Ophthalmology, 84, 378-384. http://dx.doi.org/10.1136/bjo.84.4.378

[11] Constantinou, M., Daniell, M., Snibson, G.R., Vu, H.T. and Taylor, H.R. (2007) Clinical Efficacy of Moxifloxacin in the Treatment of Bacterial Keratitis: A Randomized Clinical Trial. Ophthalmology, 114, 1622-1629. http://dx.doi.org/10.1016/j.ophtha.2006.12.011

[12] Wiley, L., Bridge, D.R., Wiley, L.A., Odom, J.V., Elliott, T. and Olson, J.C. (2012) Bacterial Biofilm Diversity in Contact Lens-Related Disease: Emerging rolE of Achromobacter, Stenotrophomonas, and Delftia. Investigative Ophthalmology \& Visual Science, 53, 3896-3905. http://dx.doi.org/10.1167/iovs.11-8762 